\title{
Isolation of Multi Drug Resistant Escherichia coli from drinking water of Lahore City, Pakistan
}

NAZIA MIR, SIDRAH SALEEM, MUHAMMAD IMRAN, AYESHA GHAZAL, MUHAMMAD USMAN

The Children Hospital, Lahore

Correspondence to Dr. Nazia Mi, WMO, Email: dr.naaziamir@gmail.com, Cell: 0324-5752042

\begin{abstract}
Background: The major faecal coliform is Escherichia coli which contaminates the drinking water from human and animal faecal waste. In developing regions drinking water is a vital source of microbiological pathogens. Multi-drug resistant (MDR) is defined as resistance to one or more antibiotic groups. An E. coli count greater than 4/dl in municipal drinking water is indicative of unacceptable fecal contamination.

Aim: To find out the frequency of MDR E. coli in water system of Lahore, Pakistan.

Methodology: Drinking water was collected from different towns of Lahore. The samples were processed using Membrane Filtration Technique. In the present study, Multidrug Resistant E.coli was isolated and antibiotic resistant pattern was seen against 16 most commonly antimicrobials, using Kirby Bauer disc diffusion method.

Results: Total 100 water samples were collected, frequency of Escherichia coliisolated was $27 \%$ and frequency of MDR E.coli was $19 \%$. The highest resistance showed by the organism towards Ampicillin (AMP) $21(81.48 \%$ ), Augmentin (AMC), and Ceftazidime (CAZ) 14(51.85\%) respectively. In this study also frequency of Extended Spectrum $\beta$-Lactamases were seen. Most common organisms isolated were E. coli $7 \%$ and Klebsiella $5 \%$. Frequency of other coliforms isolated from drinking water other than E. coli was Klebsiella species $26 \%$, Pseudomonas species 27\%, Enterobacter 7\%, Citrobacter species 8\% and Acinetobacter species 5\%.

Conclusion: This study revealed that drinking water of Lahore city is heavilycontaminated with pathogenic microorganisms. A high proportion of antibiotic resistant is due to overuse of antibiotics, in patients with mild infections and secretion of these resistant bacteria from patients to environment. One of the reasons could be the mixing of sewage lines with drinking water supply. So, there is solely requirement for further studies for the identification of the sources for these contaminants.
\end{abstract}

Keywords: Isolates, E. coli, Klebsiella, Multi-drug resistant (MDR)

\section{INTRODUCTION}

Water is an absolute necessity of life and safe drinking is the blessing of Allah. Water has the property of dissolving materials and suspended particles in its natural state so it is called universal solvent. Due to its property of natural solvent, it dissolves material and suspended particles, considered as one of the nutrients having no calories, and is an important component of diet. That's why pure and contaminants free water is mandatory for the maintenance of good health and its shortage or use of contaminated water interferes with normal physiological functions of living beings.

Pure drinking water is universally recognized as a basic human need and one of the basic or important factors for human growth as well as social and economic existence (WHO, 2007). Due to increase population acceptable water supply is decreasing and millions of people have inadequate access to clean water (Oyem et al., 2014).Contaminated drinking water by antibiotic resistant microorganism may harm and taint living individuals by antibiotic resistant pathogens consequently challenge human ability to prevent themselves from diseases. The spread of antibiotic-resistant microorganisms is causing general health problems worldwide, and marine environment is a known reservoir for antibiotic resistance genes. Various researches are conducted on antibiotic

Received on 28-10-2020

Accepted on 18-02-2021 resistance in drinking water and expressed serious concerns. A study conducted in 2014 at Guinea by Machado, A. Bordalo showed 59\% antibiotic resistance rate in drinking water(Machado \& Bordalo, 2014). Because water is integral part of human activities, so the appearance of antibiotic resistance in drinking water is of great concern for disease free livings.

Lahore is the Capital of Punjab province and second populous city of Pakistan. The population of Lahore is about 12 million and daily water requirement for inhabitants of Lahore city is approximately 400 million liters. The main source of water supply is ground water. WASA is the water supplying agency to Lahore city. The water which is supplied by WASA is not entirely pure but fecally contaminated due to poor and century old sewerage system. The drinking water should be clean from any parasites, microorganisms and other substances, which can cause danger to human health. Microbiological investigations of each tap are carried to ensure the standard of drinking water. Human and animal fecal matter are a greater risk for the contamination of drinking water whereas other sources and path of contamination can also be of significance.

Plasmids carry multiple antibiotic resistance genes, contribute to the spread of MDR. Antibiotic resistance mediated by MDR plasmids limits the treatment options for the infections caused by Gram-negative bacteria, especially family Enterobacteriaceae and Escherichia coli is one of them. These plasmids can easily be transferred to other species (Feng et al., 2002). The drinking water outbreaks of Escherichia coli are reported from various 
regions of world. From different parts of world water related gastro diseases due to Escherichia coli are reported for poor management.

Microorganisms are defined as MDR when they acquired non-susceptibility to at least one agent in three or more antimicrobial categories. (SilpiBasek et al., 2016).Surveillance data of antibiotic resistance shows that E. coli has high resistance towards ampicillin and newer antibiotics, Fluoroquinolones and Cephalosporin (Tadesse et al., 2012). An antibiotic resistance attribute in microbes is responsible for human health because it fetches severe health issues, increases risk of complications and becomes a major disease burden for hospitals. It leads to upsurge health care cost. Underground supply water is a source of contamination which are loaded by antibiotics. Therefore, the study was done to find out the frequency of MDR E. coli in water system of Lahore; Pakistan. However, there are very few studies about the presence of multi drug resistance $\mathrm{E}$. coli and its antimicrobial susceptibility pattern in local literature.

\section{MATERIAL AND METHODS}

This descriptive study was conducted at Microbiology Department, University of Health Sciences Lahore during June to October 2019. WASA is water supplying agency in Lahore city. Prior to collection of water samples from different towns of Lahore city, several visits were made at various underground water supply areas to observe the sanitary conditions of sites any source of contaminations which might affect the quality of water from the water supply. Environmental factors and presence of animal near water sample collection points were also featured. A total of 100 samples were collected in sterile autoclave glass bottles of volume capacity $300 \mathrm{ml}$ which were provided by the pharmaceutical companyOxoid UK for sample collection(Monica Cheesbrough, 2015). Tap nozzle were sterilized with $70 \%$ ethanol and adequate time of 1 to 2 minutes was given for adequate flushing of the pipelines before taking the samples. The sampling bottles were sealed and labelled with paper tape(Leiter LW et al., 1929). All Samples were transported to the laboratory for testing within 2 hours(Zareen et al., 2014) . Once in laboratory, they were stored in 4-degree centigrade temperature. Water processing was done by using Membrane Filtration Technique (MFT) and system is Millipore membrane filtration system (USA)(Monica Cheesbrough, 2015) (J.G.Collee et al,.2008).

From all 100 samples of water, $300 \mathrm{ml}$ volume of the water was filtered through a Milli pore membrane filters (47 $\mathrm{mm}$ in diameter and pore size $0.45 \mathrm{um}$ ). It is enough to retain the indicator bacteria to be counted. These membrane filters were incubated aerobically at $35^{\circ} \mathrm{C}-$ $37^{\circ} \mathrm{C}$ for $18-24$ hours on a selective indicator medium, so that the indicator bacteria grow into colonies on its upper surface. (Mackie et al., 2008). These colonies, which are recognized by their, morphology and ability to grow on the selective medium are counted. (J.G. Collee et al., 2008). Colonies were counted with the colony counter (Galaxy 230, UTECH, USA) which were formed on the surface of the MacConkey agar (Fig.1). E. coli appeared in the form of pink colored colonies on Membrane filter paper which are called lactose fermenters.

Fig.1

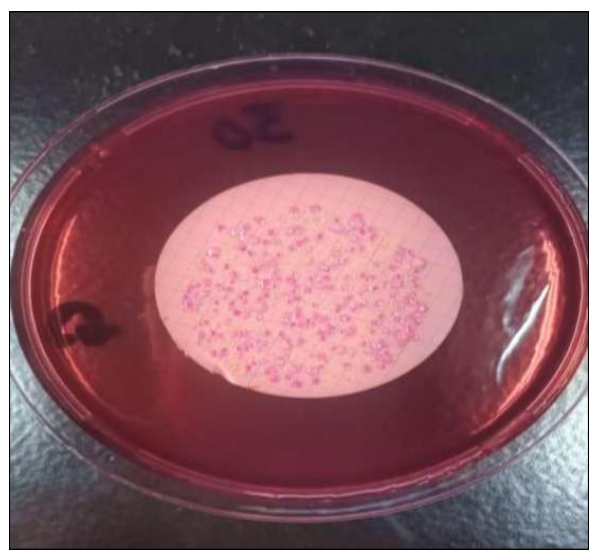

The pink colored colonies of E.coli on the Membrane filters were further identified on the basis of morphology, cultural characteristics, Gram staining, spot test and Biochemical profile (Monica Cheesbrough, June 2000). Final identification was done by API 20E (BioMerieux, France). The standard method for identification of Antibiotic sensitivity is Kirby Bauer Disc Diffusion Test(Monica Cheesbrough, 2015). E.coli strains were tested for sixteen commonly used antibiotics, i.e. Ampicillin (10ug), Amoxcillin (10ug), Ceftazidime (30ug), Cefotaxime (30ug), Ciprofloxacin (5ug), Cetriaxone(30 ug), Cefipime (30ug), Cefuroxime (30ug), Cefixime (5ug), Levofloxacin (5ug), Amikacin (30ug), Gentamicin(10 ug), Tazocin (110ug), Moxifloxacin (5ug), Imipinem (10ug), and Meropenem (10ug). (CLSI 2018). E.coli colonies were picked from MacConkey Agar plates, mixed with $1 \mathrm{ml}$ sterile saline to prepare homogenous suspension. The suspension was compared with the MacFarland turbidity standard solution. (Monica Cheesbrough, 2015). It was spread on Muller Hinton Agar with cotton swab. The antibiotic susceptibility discs were put on the agar surface with the help of sterile fine pointed needle for up at a distance of approximately $2.5 \mathrm{~cm}$ and incubated for 24 hours at $37^{\circ} \mathrm{C}$. The clear zones around the discs were noted and reported as sensitive and resistant according to CLSI guidelines 2018.

Extended spectrum $\beta$-Lactamases detection was done in vitro. E.coli colonies were taken from MacConkey plates. Colonies were mixed in $1 \mathrm{ml}$ sterile saline to prepare homogenous mixture. This homogenous mixture was compared with the MacFarland turbidity standard solution. (Monica Cheesbrough, 2015). The suspension was spread with cotton swab on Muller Hinton Agar. Antibiotic disc of Augmentin (AUG) is placed in between Cefotaxime (CTX) and Ceftazidime (CAZ). Now incubate at $37^{\circ} \mathrm{C}$ for 24 hours. Key hole pattern will be seen for ESBL production. (CLSI 2018).

Statistical analysis was done using SPSS version 25.0. The detection of organisms and antimicrobial sensitivity pattern is presented as frequency, percentages and graphs. 


\section{RESULTS}

A total of 100 tap water samples were collected from different towns of Lahore. Frequency of E. coli count was found $27 \%$ from all 100 water samples taken from different towns of Lahore city. Water samples taken from Gulberg and Data Gunj Bakhsh Town showed highest frequency of E.coli. Frequency of other coliforms isolated from drinking water other than E. coli was Klebsiella species 26\%, Pseudomonas species 27\%, Enterobacter 7\%, Citrobacter species $8 \%$ and Acinetobacter species $5 \%$ (Fig. 2). This figure showed the highest sensitivity of organism towards Imipenem (IPM) which is $96.3 \% \quad(n=26)$ followed by Meropenem (MEM) 25(92.6\%), Amikacin (AK), and Tazocin (TZP) 22(81.48\%) respectively(Fig 3) and the highest resistance shown by the organism towards Ampicillin (AMP) 21(81.48\%), Augmentin (AMC), and Ceftazidime (CAZ) 14(51.85\%) respectively (Fig. 4).

Fig.2 Frequency of Coliforms isolated from different areas of Lahore city.

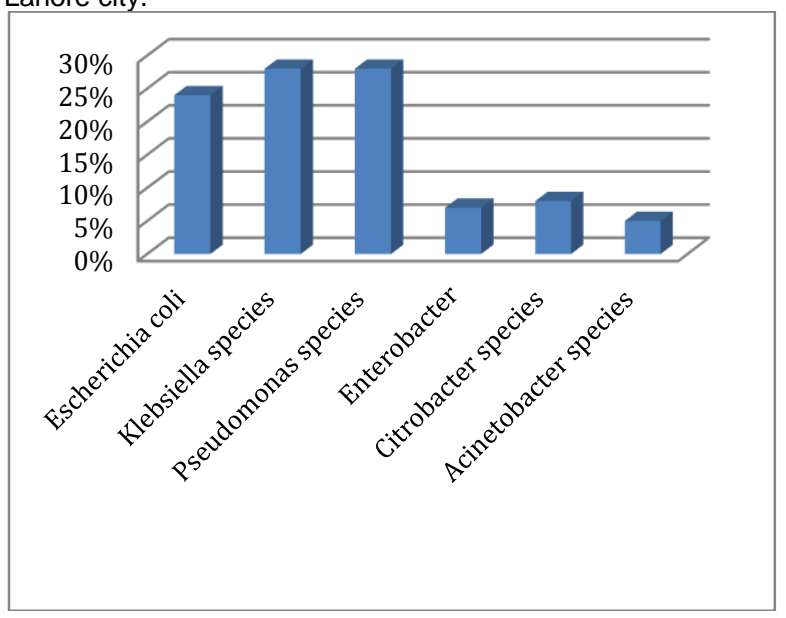

Fig. 3: E.coli sensitivity for Imipinem and Meropenem

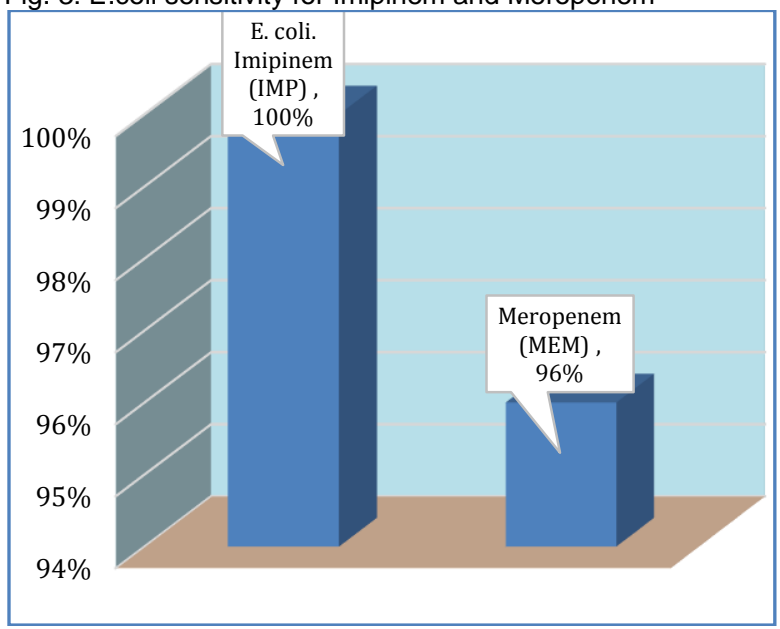

Sensitivity pattern of Klebsiella specie showed the highest sensitivity towards Ciprofloxacin, Levofloxacin, Gentamicin, Imipinem followed by Meropenem, Amikacin and Cefuroxime. Ampicillin shows the highest resistant antibiotic followed by Cefipime, Ceftazidime and
Amoxicillin. Pseudomonas species showed the highest sensitivity pattern towards Cefuroxime, Levofloxacin, Amikacin, Meropenem followed by Gentamicin, Ciprofloxacin and Imipinem, whereas Amoxacillin shows the highest resistant antibiotic followed by Cefotaxime and Ceftazidime

Fig. 4: Resistant pattern of E. coli towards Ampicillin and Ceftazidime

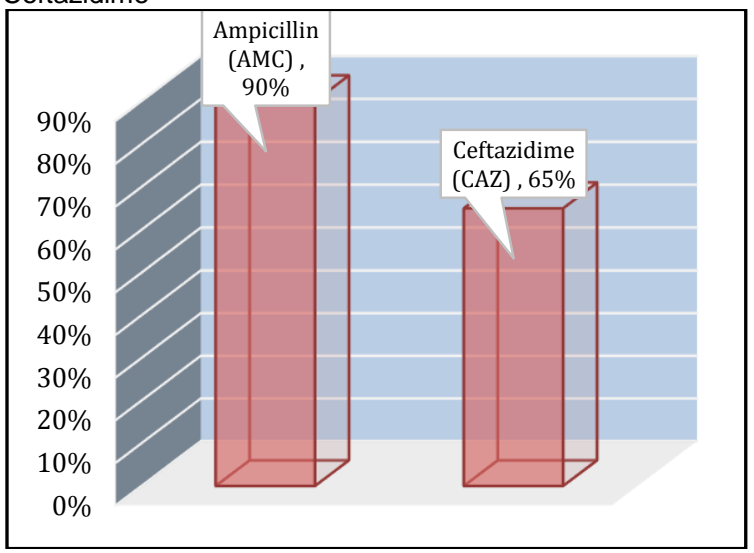

\section{DISCUSSION}

Safe water supply is necessary for the health of every individual. Pure and safe drinking water is still inadequate in the world. Drinking water is suitable for use if it is clean physically, chemically and biologically, so that it can be safely used for drinking and cooking(Zareen et al., 2014). Globally, waves of food borne and water diseases epidemics have been reported(Wood et al., 2010). Due to inadequate water treatment and compromise with the distribution system and is a great threat by pathogenic microorganism(Azizullah et al., 2011). Water borne diseases are causing serious problems e.g, diarrhea, cholera, dysentery and there is also increase threat of antibiotic resistant pathogens. Researchers are focused on the natural environment for antibiotic resistance(Wellington et al., 2013). Bacterial resistant can be innate (characteristics of particular species) or acquired. In this study drinking water samples were taken from tap water of homes of different areas of Lahore city. Multidrug resistant Escherichia coli were isolated from a total of 100 water samples. Multi drug resistance was labelled when it was resistant to more than two antibiotics (Magiorakos et al., 2012). Frequency of E.coli from different areas was $24 \%$. This finding is similar with the other studies where $71 \%$ E.coli isolated from drinking water out of 155 samples(Lyimo et al., 2016). Nidhi $K$ studied the Prevalence of pathogenic bacteria on fecal coliforms. 50 water samples were taken, E.coli isolated from theses samples were 12(Sood et al., 2015). This result in the study revealed that water is heavily contaminated with faecal matter and E.coli is a member of faecal coliform and contaminates the drinking water from animal faecal wastes. In this study detection of MDR E. coli was done by Kirby Bauer disc diffusion method, which is standard method for the detection of sensitive and resistant antibiotics for the organism.(Monica Cheesbrough, 2015). 
A local same study done in Aga Khan University Karachi, Pakistan where 42 water samples were collected out of which $62.96 \% \mathrm{E}$. coli isolated exhibited resistance against three or more antibiotics, $25.92 \%$ organism were resistant to three antibiotics, and $11.11 \%$ were determined to be resistant to six antimicrobials. Ampicillin shows the highest resistant i.e., $88.89 \%$ (Patoli et al., 2010). A same study was done by Nidhi K Sood et al (2015) in India on watersamples in which overall most frequently observed resistant towards antibiotic was ampicillin. Another study shows the resistant to Penicillin group (94.95\%). 50\% organisms were resistant to ampicillin and amoxicillin(Nazir et al., 2005). Broad spectrum antimicrobial resistant organisms are now widely emerging in hospital environment causing serious infection in immunocompetent hosts andE.coli is well known organism for the antibiotic resistant profile. (Nidhi K Soodet al., 2015). Gram negative bacteria have emerging resistance to penicillin due to the production of $\beta$-lactamases group of antimicrobials (Islam et al., 2018).

In this study MDREscherichia coli was isolated from residential areas of Lahore city, Pakistan. In this study, isolation of resistant strain of $\mathrm{E}$. coli in domestic water shows the importance of regular surveillance by water and sanitary authority, and make sure the possible cleaning of water system. In fact, emergence of multidrug resistance in Escherichia coli is the matter of concerns which makes the treatment difficult in case of water borne diseases. The further studies could be found to analyze the mechanism of exposure of bacteria to antibiotics and spreading of antibiotic resistant bacteria in different fresh water environment.

\section{RECOMMENDATIONS}

A high-level study should be conducted on water with microbial and chemical analysis of drinking water of Lahore.

Genetic sequencing of resistant E. coli strains should be performed to find the origin of these bacteria.

A study should be conducted with the prevalence of shiga toxin gene in Multi drug resistant $\mathrm{E}$. coli.

\section{REFERENCES}

1. Azizullah, A., Khattak, M. N. K., Richter, P., \& Häder, D. P. (2011). Water pollution in Pakistan and its impact on public health - A review.

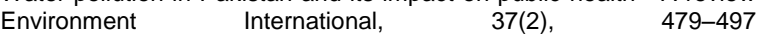
https://doi.org/10.1016/j.envint.2010.10.007

2. Islam, K., Jashimuddin, M., Nath, B., \& Nath, T. K. (2018). Land use classification and change detection by using multi-temporal remotely sensed imagery: The case of Chunati wildlife sanctuary, Bangladesh. Egyptian Journal of Remote Sensing and Space Science, 21(1), 3747. https://doi.org/10.1016/j.ejrs.2016.12.005

3. Leiter LW 1929. The Eijkman Fermentation Test as an Aid in the Detction of Faecal organisms in Water. Amer J Hygiene. 9, 705 - 24

4. Lyimo, B., Buza, J., Subbiah, M., Smith, W., \& Call, D. R. (2016). Comparison of antibiotic resistant Escherichia coli obtained from drinking water sources in northern Tanzania: a cross-sectional study.
BMC Microbiology. https://doi.org/10.1186/s12866-016-0870-9

5. J. Gerald Collee, Barie P Marnion, Andrew G. Fraser, Anthony Simmons. Mackie and MacCartney Practical Medical Microbiology $14^{\text {th }}$ Ed,2008; PP 887-888

6. K. H. M. N. H. N., . M. B. R., . K. M. N., F. A., . M. F. R. K., \&. M. S. I. (2005). Antibiotic Sensitivity of Escherichia coli Isolated from Water and its Relation with Plasmid Profile Analysis. Pakistan Journal of Biological Sciences, 8(11), 1610-1613. https://doi.org/10.3923/pjbs.2005.1610.1613

7. Machado, A., \& Bordalo, A. A. (2014). Prevalence of antibiotic resistance in bacteria isolated from drinking well water available in Guinea-Bissau (West Africa). Ecotoxicology and Environmental Safety. https://doi.org/10.1016/j.ecoenv.2014.04.037

8. Magiorakos, A. P., Srinivasan, A., Carey, R. B., Carmeli, Y., Falagas, M. E., Giske, C. G., Harbarth, S., Hindler, J. F., Kahlmeter, G., OlssonLiljequist, B., Paterson, D. L., Rice, L. B., Stelling, J., Struelens, M. J., Vatopoulos, A., Weber, J. T., \& Monnet, D. L. (2012). Multidrugresistant, extensively drug-resistant and pandrug-resistant bacteria: An international expert proposal for interim standard definitions for acquired resistance. Clinical Microbiology and Infection. https://doi.org/10.1111/j.1469-0691.2011.03570.x

9. Monica Cheesbrough (editor). Biochemical tests to identify bacteria. In: District laboratory Practice in tropical countries part 2. Cambridge University Press. 2000:pg62-70.

10. Monica Cheesbrough. (2015). Biochemical tests to identify bacteria. In: District laboratory Practice in tropical countries part 2 (Second). Cambridge University Press.

11. Oyem, H. ., Oyem, I. ., \& Ezeweali, D. (2014). Temperature, pH, EC, TDS, COD in grorundwater. Research Journal of Environmental Sciences, 8(3), 271-275. http://dx.doi.org/10.3168/jds.20147912\%5Cnhttp://dx.doi.org/10.1016/j.jchromb.2010.11.010\%5Cnhttp:// dx.doi.org/10.1016/j.carbpol.2010.05.029

12. P. Feng, S. D. Weagant, M. A. Grant and W. Burkhardt, "2002, Chapter 4. Enumeration of Escherichia coli and the Coliform Bacteria," In: Food and Drug Administration (FDA), Bacteriological Analytical Manual Online, 8th Edition, Silver Spring, Berlin, 1998.

13. Patoli, A. A., Patoli, B. B., \& Mehraj, V. (2010). High Prevalence of Multi-Drug Resistant Escherichia coli in Drinking Water Samples from Hyderabad. Gomal Journal of Medical Sciences, 8(1), 23-26.

14. Silpi, B., Priyanka, S, and Monali, R.2016. Multidrug-Resistant and Extensively Drug-resistant Bacteria. J. Patho.2016. https://www.hindawi.com/journals/jpath/2016/4065603

15. Sood, N., Patel, P., Patel, S., \& Mandalia, A. (2015). A study of prevalence of pathogenic bacteria, particularly, fecal coliforms and their antibiotic resistance pattern in environmental water samples of a tertiary-care hospital, Ahmedabad. International Journal of Medical Science and Public Health. https://doi.org/10.5455/ijmsph.2015.10042015359

16. Tadesse, D. A., Zhao, S., Tong, E., Ayers, S., Singh, A., Bartholomew, M. J., \& McDermott, P. F. (2012). Antimicrobial drug resistance in Escherichia coli from humans and food animals, United States, 1950 2002. Emerging Infectious Diseases, 18(5), 741-749. https://doi.org/10.3201/eid1805.111153

17. Wellington, E. M. H., Boxall, A. B. A., Cross, P., Feil, E. J., Gaze, W H., Hawkey, P. M., Johnson-Rollings, A. S., Jones, D. L., Lee, N. M., Otten, W., Thomas, C. M., \& Williams, A. P. (2013). The role of the natural environment in the emergence of antibiotic resistance in Gramnegative bacteria. The Lancet Infectious Diseases, 13(2), 155-165. https://doi.org/10.1016/S1473-3099(12)70317-1

18. WHO. (2007). Combating waterborne disease at the household level / International Network to Promote Household Water Treatmente and Safe Storage. World Health Organization, 1-13.

19. Wood, J. D., Bezanson, G. S., Gordon, R. J., \& Jamieson, R. (2010). Population dynamics of Escherichia coli inoculated by irrigation into the phyllosphere of spinach grown under commercial production conditions. International Journal of Food Microbiology, 143(3), 198204. https://doi.org/10.1016/j.ijfoodmicro.2010.08.022

20. Zareen, M., Sajid, I., \& Ali, B. (2014). Isolation and detection of Escherichia coli O157 from potable water system of Lahore, Pakistan. Pakistan Journal of Zoology. 